\title{
Gambling in contests
}

Citation for published version (APA):

Seel, C., \& Strack, P. (2013). Gambling in contests. Journal of Economic Theory, 148(5), 2033-2048. https://doi.org/10.1016/j.jet.2013.07.005

Document status and date:

Published: 01/01/2013

DOI:

10.1016/j.jet.2013.07.005

Document Version:

Publisher's PDF, also known as Version of record

Document license:
Taverne

\section{Please check the document version of this publication:}

- A submitted manuscript is the version of the article upon submission and before peer-review. There can be important differences between the submitted version and the official published version of record.

People interested in the research are advised to contact the author for the final version of the publication, or visit the DOI to the publisher's website.

- The final author version and the galley proof are versions of the publication after peer review.

- The final published version features the final layout of the paper including the volume, issue and page numbers.

Link to publication

\footnotetext{
General rights rights.

- You may freely distribute the URL identifying the publication in the public portal. please follow below link for the End User Agreement:

www.umlib.nl/taverne-license

Take down policy

If you believe that this document breaches copyright please contact us at:

repository@maastrichtuniversity.nl

providing details and we will investigate your claim.
}

Copyright and moral rights for the publications made accessible in the public portal are retained by the authors and/or other copyright owners and it is a condition of accessing publications that users recognise and abide by the legal requirements associated with these

- Users may download and print one copy of any publication from the public portal for the purpose of private study or research.

- You may not further distribute the material or use it for any profit-making activity or commercial gain

If the publication is distributed under the terms of Article $25 \mathrm{fa}$ of the Dutch Copyright Act, indicated by the "Taverne" license above, 


\title{
Gambling in contests
}

\author{
Christian Seel $^{\mathrm{a}, *}$, Philipp Strack ${ }^{\mathrm{b}}$ \\ a Maastricht University, School of Business and Economics, Department of Economics, P.O. Box 616, 6211 Maastricht, \\ The Netherlands \\ ${ }^{\mathrm{b}}$ University of Bonn, Department of Economics, Germany
}

Received 11 September 2009; final version received 15 March 2013; accepted 2 May 2013

Available online 25 July 2013

\begin{abstract}
This paper presents a strategic model of risk-taking behavior in contests. Formally, we analyze an $n$-player winner-take-all contest in which each player decides when to stop a privately observed Brownian motion with drift. A player whose process reaches zero has to stop. The player with the highest stopping point wins. Unlike the explicit cost for a higher stopping time in a war of attrition, here, higher stopping times are riskier, because players can go bankrupt. We derive a closed-form solution of a Nash equilibrium outcome. In equilibrium, highest expected losses occur at an intermediate negative value of the drift.
\end{abstract}

(c) 2013 Elsevier Inc. All rights reserved.

JEL classification: C72; C73; D81

Keywords: Discontinuous games; Contests; Relative performance pay; Risk-taking behavior

\section{Introduction}

To provide more excitement for the players, the (online) gambling industry introduced casino tournaments. The rules are simple: all participants pay a fixed amount of money prior to the tournament - the "buy-in" - that enters into the prize pool. In return, they receive chips, which they can invest in the casino gamble throughout the tournament. At the end of the tournament, the player who has most chips wins a prize, which is the sum of the buy-ins minus some fee charged by the organizers. Benefits are two-sided: players restrict their maximal loss to the buy-in and

\footnotetext{
* Corresponding author.

E-mail addresses: c.seel@maastrichtuniversity.nl (C. Seel), philipp.strack@uni-bonn.de (P. Strack).
} 
enjoy a new, strategic component of the game; the casino makes a sure profit through the fee it charges.

The observability of each other's chip stacks throughout the tournament depends on the provider. The no-observability case is a good illustration of our model-in equilibrium, players use the gamble even though it has a negative expected value.

In the model, each player decides when to stop a privately observed Brownian motion with (usually negative) constant drift, constant variance, and positive initial value. If a player becomes bankrupt, i.e., the Brownian motion hits zero, she has to stop. The player who stops her process at the highest value wins a prize.

In equilibrium, players maximize their winning probability rather than the expected value of the process. Hence, they do not stop immediately even if the underlying process is decreasing in expectation. Intuitively, if all other players stop immediately, it is better for the remaining player to play until she wins a small positive amount or goes bankrupt, since she can ensure she wins an arbitrarily small positive amount with a probability arbitrarily close to one.

In the unique equilibrium outcome for two players, expected losses are non-monotonic in the expected change of the process per time-a larger drift can lead to higher expected losses. Intuitively, for an only slightly negative drift, players have a relatively high probability of obtaining a gain from continuation. In equilibrium, this makes players stop later than for a larger negative value of the drift. Thus, an increase in the drift leads to lower expected losses per time, but it also increases the equilibrium stopping time. Our calculation shows that expected losses are maximal for an intermediate negative value of the drift. If a third party-e.g., a contest designer who might have imperfect information about the drift—obtains gains or losses of the players, contests are not a reliable compensation scheme, because even with a slightly negative drift, the third party might incur a large loss.

The formal analysis proceeds as follows. Proposition 1 derives a formula for an implied stopping chance in a symmetric equilibrium of the $n$-player contest that pinpoints a candidate equilibrium distribution. To do so, we suppose that each player is indifferent between stopping and continuing as long as the value of the process remains in a certain interval.

For the two-player case, Proposition 2 derives an equilibrium stopping time that induces the candidate equilibrium distribution explicitly. The equilibrium strategy mixes over strategies that stop the first time the process leaves a fixed interval. Proposition 3 shows uniqueness of the equilibrium distribution.

For more than two players, Proposition 4 ensures the existence of a stopping time that induces the candidate equilibrium distribution. Its proof relies on a result in probability theory on the Skorokhod embedding problem. This literature—initiated by Skorokhod [17,18]—analyzes the conditions under which a stopping time of a stochastic process exists that embeds, i.e., induces, a given probability distribution; for an excellent survey article, see Oblój [13]. In the proof of Proposition 4, we verify a sufficient condition from Pedersen and Peskir [15]. In Proposition 5 and Proposition 6, we extend the previous construction to two players with asymmetric diffusion parameters. This whole approach is new to game theory, and the main technical contribution of this paper.

Proposition 7 provides the main characterization result: the general shape of the expected value of the stopped processes is quasi-convex in drift and variance, falling and then rising. In particular, highest expected losses occur at an intermediate negative value of the drift.

Apart from casino tournaments, this paper provides a stylized model for the following applications. First, consider a private equity fund that invests in start-up companies. The value of the fund is mostly private information until maturity, because start-ups do not trade on the stock 
market and the composition of the fund is often unknown. The model analyzes a competition between fund managers in which, at maturity, the best performing manager gets a prize-a bonus or a job promotion.

In this application, there are several possible reasons for a downward drift. For instance, there may be no good investment opportunities in the market. Moreover, the downward drift may capture the cost of paying an expert to search for possible investments. The model predicts that the return on investment is very sensitive to the profitability of investment opportunities. In particular, an intermediate negative drift is most harmful for the investors. In this case, fund managers who compete in a contest behave as if they were risk-loving, which a payment based on absolute success could avoid.

As a second example, consider a competition in a declining industry. In a duopoly, for instance, firms compete to survive and get the monopoly profit. Fudenberg and Tirole [5] model the situation as a war of attrition-only the firm who stays alone in the market wins a prize, but both incur costs until one firm drops out.

In an interpretation of our model, managers of both firms decide if they want to make risky investments - into R\&D or stocks of other firms. Investments are costly, but could improve the firm's value. When the duopoly becomes unprofitable, the firm with the higher value winseither by a take-over battle or because the other firm cannot compete in a price war-and its manager keeps his job.

Our model predicts that managers choose very risky strategies. In particular, investors lose most money in expectation if investment opportunities have an intermediate negative expected value, which is consistent with being in a declining industry. This effect increases in the asymmetry of the firms' values. Intuitively, to satisfy the indifference condition for the stronger firm, the weaker firm has to make up for its initial disadvantage by taking higher risks.

\section{Related literature}

Hvide [9] investigates whether tournaments lead to excessive risk-taking behavior. He modifies Lazear and Rosen [11] by assuming that players bear costs to raise their expected value, but can raise their variance without costs. In equilibrium, they choose maximum variance and low effort. Similarly, Anderson and Cabral [1] scrutinize an infinite competition in which two players, who observe each other, can update their binary choice of variance continuously. In their model, flow payoffs depend on the difference in contest success. In equilibrium, both players choose the risky strategy until the lead of one player is above a threshold; in this case, the leader switches to the save option.

In the literature on races, players balance a higher effort cost against a higher winning probability. Moscarini and Smith [12]—building on a discrete time model of Harris and Vickers [7]-analyze a two-person continuous-time race with costly effort choice. In equilibrium, effort is increasing in the lead of a player up to some threshold above which the laggard resigns; for an application to political economy, see also Gul and Pesendorfer [6]. These papers assume full observability of each other's contest success over time. In our model, however, stopping decisions and realizations of the rivals are unobservable.

Regarding the assumptions on information and payoffs, the model most resembles a silent timing game - as first explored in Karlin [10], and most recently, in Park and Smith [14]. The latter paper also generalizes the all-pay war of attrition, and so assumes that later stopping times cost linearly more. Contrary to a silent timing game, in the present paper, players do not only possess private information about their stopping decision, but also about the realization of their stochastic process. 
Finally, the paper relates to the finance literature on gambling for resurrection; e.g., Downs and Rocke [4]. In this literature, managers take unfavorable gambles for a chance to save their firms from bankruptcy. Here, however, players take high risks to veil their contest outcomes.

We proceed as follows. Section 2 introduces the model. Section 3 derives the equilibrium distribution. Section 4 extends the previous construction to the asymmetric two-player case. In Section 5, we state the main characterization result, Proposition 7, and discuss its implications. Section 6 concludes. We relegate several proofs to Appendix A.

\section{The model}

There are $n$ agents $i \in\{1,2, \ldots, n\}=N$ who face a stopping problem in continuous time. At each point in time $t \in \mathbb{R}_{+}$, agent $i$ privately observes the realization of a stochastic process $X^{i}=\left(X_{t}^{i}\right)_{t \in \mathbb{R}_{+}}$with

$$
X_{t}^{i}=x_{0}+\mu t+\sigma B_{t}^{i} .
$$

The constant $x_{0}>0$ denotes the starting value of all processes. The drift $\mu \in \mathbb{R}$ is the common expected change of each process $X_{t}^{i}$ per time, i.e., $\mathbb{E}\left(X_{t+\Delta}^{i}-X_{t}^{i}\right)=\mu \Delta$. The noise term is an $n$-dimensional Brownian motion $B_{t}$ scaled by $\sigma>0$. In Section 4 , we allow for heterogeneity in all parameters and derive an equilibrium for the two-player case.

A strategy of player $i$ is a stopping time $\tau^{i}$. This stopping time depends only on the realization of his process $X^{i}$, as the player only observes his own process. ${ }^{1}$ Mathematically, the agents' stopping decision until time $t$ has to be $\mathcal{F}_{t}^{i}$-measurable, where $\mathcal{F}_{t}^{i}=\sigma\left(\left\{X_{s}^{i}: s<t\right\}\right)$ is the sigma-algebra induced by the possible observations of the process $X^{i}$ before time $t$. We restrict agents' strategy spaces in two ways. First, we require finite expected stopping times, i.e., $\mathbb{E}\left(\tau^{i}\right)<\infty$. Second, a player has to stop in case of bankruptcy. More formally, we require $\tau^{i} \leqslant \inf \left\{t \in \mathbb{R}_{+}: X_{t}^{i}=0\right\}$ a.s.. To incorporate mixed strategies, we allow for randomized stopping times-progressively measurable functions $\tau^{i}(\cdot)$ such that for every $r^{i} \in[0,1], \tau^{i}\left(r^{i}\right)$ is a stopping time. Intuitively, agents can draw a random number $r^{i}$ from the uniform distribution on $[0,1]$ before the game and play a stopping strategy $\tau^{i}\left(r^{i}\right)$.

The player who stops his process at the highest value wins a prize, which we normalize to one without loss of generality. Ties are broken randomly. Formally,

$$
\pi^{i}=\frac{1}{k} \mathbf{1}_{\left\{X_{\tau^{i}}^{i}=\max _{j \in N} X_{\tau^{j}}^{j}\right\}},
$$

where $k=\left|\left\{i \in N: X_{\tau^{i}}^{i}=\max _{j \in N} X_{\tau^{j}}^{j}\right\}\right|$. Hence, the game is a constant sum game. All agents maximize their expected payoff, i.e., the probability of winning the contest. This optimization is independent of their risk attitude.

To ensure equilibrium existence in finite time stopping strategies, we henceforth impose a technical condition that places a positive upper bound on $\mu$ :

Assumption 1. $\mu<\log \left(1+\frac{1}{n-1}\right) \frac{\sigma^{2}}{2 x_{0}}$.

\footnotetext{
1 The equilibrium of the model would be the same if the stopping decision was reversible and stopped processes were constant.
} 


\section{Equilibrium analysis}

In this section, we first derive a candidate equilibrium distribution. We then prove that there exists a stopping time inducing the candidate equilibrium distribution and show uniqueness of the equilibrium distribution for the two-player case.

\subsection{The equilibrium distribution}

Every strategy of agent $i$ induces a (potentially non-smooth) cumulative distribution function (cdf) $F_{i}: \mathbb{R}_{+} \rightarrow[0,1]$ of his stopped process, where $F_{i}(x)=\mathbb{P}\left(X_{\tau^{i}}^{i} \leqslant x\right)$. Denote the right endpoint of the support of the distribution of player $i$ by $\bar{x}^{i}=\sup \left\{x: F_{i}(x)<1\right\}$ and $\bar{x}=\max _{i} \bar{x}^{i}$. Denote the winning probability of player $i$ if he stops at $X_{\tau^{i}}^{i}=x$ by $u_{i}(x)$, where

$$
u_{i}(x)=\mathbb{P}\left(x>\max _{j \neq i} X_{\tau^{j}}^{j}\right)+\mathbb{E}\left(\frac{1}{k} \mid x=X_{\tau^{i}}^{i}=\max _{j \neq i} X_{\tau^{j}}^{j}\right) \mathbb{P}\left(x=\max _{j \neq i} X_{\tau^{j}}^{j}\right) .
$$

If no distribution contains a mass point, for all $x$, we get

$$
u_{i}(x)=\prod_{j \neq i} \mathbb{P}\left(X_{\tau^{j}}^{j} \leqslant x\right)=\prod_{j \neq i} F_{j}(x) .
$$

In equilibrium, each player maximizes his winning probability $\mathbb{E}\left(u_{i}\left(X_{\tau^{i}}^{i}\right)\right)$ over strategies $\tau^{i}$. In the following, we derive an equilibrium distribution in which no player places a mass point and which is identical across players. Hence, for notational convenience, we suppress subscripts and superscripts $i$. Denote by $\tau_{(a, b)}=\inf \left\{t: X_{t} \notin(a, b)\right\}$ the first leaving time of the set $(a, b)$. The probability of hitting the upper barrier $b$ for Brownian motion with two absorbing barriers is

$$
\rho(a, b, x)=\mathbb{P}\left(X_{\tau_{(a, b)}}=b \mid x_{0}=x\right)=\frac{\exp \left(\frac{-2 \mu a}{\sigma^{2}}\right)-\exp \left(\frac{-2 \mu x}{\sigma^{2}}\right)}{\exp \left(\frac{-2 \mu a}{\sigma^{2}}\right)-\exp \left(\frac{-2 \mu b}{\sigma^{2}}\right)},
$$

for all $x \in(a, b)$. We derive an equilibrium such that, for any point $x \in(0, \bar{x})$, a player is indifferent between stopping at $x$ and continuing with the strategy $\tau_{(0, \bar{x})}$. From Eq. (3), we obtain

$$
u(x)=\rho(0, \bar{x}, x) u(\bar{x})+(1-\rho(0, \bar{x}, x)) u(0)=\rho(0, \bar{x}, x)
$$

for all $x \in(0, \bar{x})$. Intuitively, Eq. (4) illustrates the trade-off between a higher stopping value (here $\bar{x}$ ) and a higher risk of bankruptcy. By symmetry and optimality of stopping at $x_{0}$, we get $u\left(x_{0}\right)=\frac{1}{n}=\rho\left(0, \bar{x}, x_{0}\right)$ which uniquely fixes $\bar{x}$ as

$$
\bar{x}=-\frac{\sigma^{2}}{2 \mu} \log \left(n\left(\exp \left(\frac{-2 \mu x_{0}}{\sigma^{2}}\right)-1\right)+1\right) .
$$

Note that $\bar{x}<\infty$ if and only if Assumption 1 holds. Intuitively, if the drift becomes too large, for every point $x$, the strategy which stops only at 0 and $x$ reaches $x$ with a probability higher than $\frac{1}{n}$.

Inserting $\bar{x}$ and $\rho(0, \bar{x}, x)$ yields $u(x)$ as

$$
u(x)=\min \left\{1, \frac{1}{n} \frac{\exp \left(\frac{-2 \mu x}{\sigma^{2}}\right)-1}{\exp \left(\frac{-2 \mu x_{0}}{\sigma^{2}}\right)-1}\right\} .
$$

Using symmetry in Eq. (2), we get $F(x)=\sqrt[n-1]{u(x)}$. Hence, we characterize a candidate for an equilibrium distribution as follows (for an illustration, see Fig. 1): 


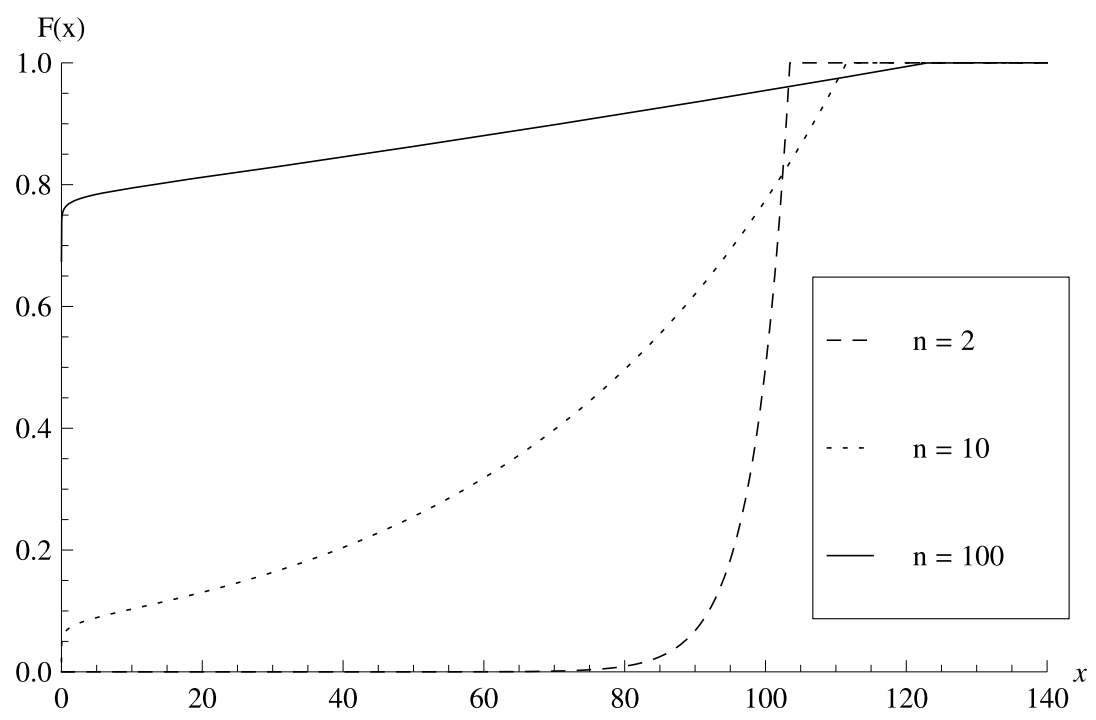

Fig. 1. An example $\left(\mu=-0.1, x_{0}=100, \sigma=1\right)$ of the equilibrium cdf's for different number of players $n$.

Proposition 1 (Equilibrium distribution). Assume $\mu \neq 0$. A strategy profile is a Nash equilibrium, if each player's strategy induces the cumulative distribution function

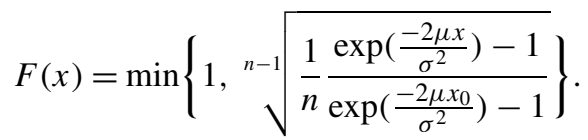

Proof. By symmetry, each player wins with probability $\frac{1}{n}$ if his strategy induces the distribution $F$. We need to show that no deviation gives a player a winning probability greater than $\frac{1}{n}$. Note that, by construction of the function $F$, the process $\left(u\left(X_{t}\right)\right)_{t \in \mathbb{R}_{+}}$is a supermartingale. For every stopping time $\tau<\infty$, consider the sequence of bounded stopping times $\min \{\tau, m\}$ for $m \in \mathbb{N}$. By Doob's optional stopping theorem (Revuz and Yor [16, p. 70]), $\mathbb{E}\left(u\left(X_{\min \{\tau, m\}}\right)\right) \leqslant$ $u\left(X_{0}\right)$. As $u\left(X_{t}\right) \in[0,1]$ is bounded, we can apply the dominated convergence theorem to get

$$
\mathbb{E}\left(u\left(X_{\tau}\right)\right)=\mathbb{E}\left(\lim _{m \rightarrow \infty} u\left(X_{\min \{\tau, m\}}\right)\right)=\lim _{m \rightarrow \infty} \mathbb{E}\left(u\left(X_{\min \{\tau, m\}}\right)\right) \leqslant u\left(X_{0}\right)=\frac{1}{n} .
$$

Intuitively, for a negative drift, each player's winning probability is convex in the stopping value on the support of the candidate equilibrium distribution. Hence, players would prefer a mean-preserving spread to a sure outcome. In the equilibrium construction, the negative effect, i.e., expected losses from waiting longer, balances out with the positive effect of a higher variance through a higher stopping time. Therefore, players are indifferent whether to stop as long as the process remains in the interval $(0, \bar{x})$.

For a consistency test on our analysis, let us consider the special case in which $X_{t}$ is a martingale, i.e., $\mu=0$. The same calculation as in the case $\mu \neq 0$ yields a candidate for an equilibrium distribution, where

$$
F(x)=\min \left\{1, \sqrt[n-1]{\frac{x}{n x_{0}}}\right\} .
$$


$F(x)$ is continuous in $\mu$ at $\mu=0$, because the same formula follows by taking limits in Eq. (5), using the approximation $e^{A}=1+A+O\left(A^{2}\right)$ for small $A$.

\subsection{Equilibrium strategies}

So far, we have been silent about the existence of a finite time stopping strategy $\tau$ inducing the candidate equilibrium distribution $F$ defined in Eq. (5). In the next step, we explicitly derive an equilibrium strategy for the two-player case. This strategy is a mixture over deterministic threshold strategies. Although we do not use the explicit strategy in the remainder of the paper, we construct it here to provide an example of equilibrium behavior. For this purpose, we introduce the transformation $\phi: \mathbb{R}_{+} \rightarrow \mathbb{R}_{+}$, where

$$
\phi(x)=\frac{\exp \left(\frac{-2 \mu x}{\sigma^{2}}\right)-1}{\exp \left(\frac{-2 \mu x_{0}}{\sigma^{2}}\right)-1} .
$$

For any twice differentiable function $\Phi: \mathbb{R} \rightarrow \mathbb{R}$, Itô's lemma characterizes the infinitesimal change of the process $\left(\Phi\left(X_{t}\right)\right)_{t \in \mathbb{R}_{+}}$as

$$
\mathrm{d} \Phi\left(X_{t}\right)=\left(\mu \Phi^{\prime}\left(X_{t}\right)+\frac{\sigma^{2}}{2} \Phi^{\prime \prime}\left(X_{t}\right)\right) \mathrm{d} t+\sigma \Phi^{\prime}\left(X_{t}\right) \mathrm{d} B_{t} .
$$

Since $\mu \phi^{\prime}(x)-\frac{\sigma^{2}}{2} \phi^{\prime \prime}(x)=0$, the process $\left(\phi\left(X_{t}\right)\right)_{t \in \mathbb{R}_{+}}$is a martingale by Eq. (7). Note that, in the two-player case, $F(x)=\phi(x) / 2$ for all $x \in[0, \bar{x}]$.

Proposition 2 (Equilibrium strategy for two players). If an agent randomly selects a number $\alpha$ from the uniform distribution on $(0,1]$ and stops if

$$
\tau=\inf \left\{t \geqslant 0:\left|\phi\left(X_{t}\right)-1\right| \geqslant \alpha\right\},
$$

then the cumulative distribution function induced by this strategy equals $F$ defined in Eq. (5) with $n=2$, i.e., $\mathbb{P}\left(X_{\tau} \leqslant x\right)=F(x)$.

Proof. By the martingale property of $\phi\left(X_{t}\right)_{t \in \mathbb{R}_{+}}$, we get

$$
\mathbb{P}\left(\phi\left(X_{\tau}\right)=1-\alpha\right)=\mathbb{P}\left(\phi\left(X_{\tau}\right)=1+\alpha\right)=\frac{1}{2} .
$$

As $\alpha$ is uniformly distributed on $(0,1]$ and the agent stops iff $\phi\left(X_{t}\right)=1 \pm \alpha$, the random variable $\phi\left(X_{\tau}\right)$ is uniformly distributed on $[0,2]$. It follows that

$$
\mathbb{P}\left(X_{\tau} \leqslant x\right)=\mathbb{P}\left(\phi\left(X_{\tau}\right) \leqslant \phi(x)\right)=\frac{\phi(x)}{2}=F(x) .
$$

Hence, we have found a strategy which induces the distribution $F(x)$ defined in Eq. (5) for the two-player case. By Proposition 1, this strategy is an equilibrium strategy. In the next step, we also establish uniqueness of the equilibrium distribution for the two-player case. The proof combines our construction with well-known results about two-player constant sum games.

Proposition 3 (Uniqueness of the equilibrium distribution for two players). In any Nash equilibrium, both players use a strategy inducing the distribution $F$ defined in Eq. (5) with $n=2$. 
For more than two players, we now prove feasibility of the equilibrium distribution (5), i.e., the existence of a stopping time which yields the target distribution. The proof requires an auxiliary result from probability theory on the Skorokhod embedding problem. This literature studies whether a distribution is feasible by stopping a stochastic process; in their terminology, there exists an embedding of a probability distribution in the process. Skorokhod $[17,18]$ analyzes the problem of embedding in Brownian motion without drift. In a recent contribution, Pedersen and Peskir [15] derive a necessary and sufficient condition for non-singular diffusions. ${ }^{2}$ They define the scale function $S$ by $^{3}$

$$
S(x)=-\frac{\sigma^{2}}{2 \mu}\left(\exp \left(\frac{-2 \mu x}{\sigma^{2}}\right)-1\right) .
$$

Lemma 1. (Pedersen and Peskir [15, Theorem 2.1].) Let $\left(X_{t}\right)$ be a non-singular diffusion on $\mathbb{R}$ starting at zero, let $S(\cdot)$ denote its scale function satisfying $S(0)=0$, and let $v$ be a probability measure on $\mathbb{R}$ satisfying $|S(x)| v(\mathrm{~d} x)<\infty$. Set $m=\int_{\mathbb{R}} S(x) v(\mathrm{~d} x)$. Then there exists a stopping time $\tau_{*}$ for $\left(X_{t}\right)$ such that $X_{\tau_{*}} \sim v$ if and only if one of the following four cases holds:

1. $S(-\infty)=-\infty$ and $S(\infty)=\infty$;

2. $S(-\infty)=-\infty, S(\infty)<\infty$ and $m \geqslant 0$;

3. $S(-\infty)>-\infty, S(\infty)=\infty$ and $m \leqslant 0$;

4. $S(-\infty)>-\infty, S(\infty)<\infty$ and $m=0$.

The values of $S(-\infty)$ and $S(\infty)$ in the previous lemma uniquely characterize the feasibility condition on $m$. For case 1 , there exists a stopping time for arbitrary $m$, while for cases $2-4$, $m=0$ is a sufficient condition. Therefore, it suffices to show $m=0$, which we do in the proof of the following proposition. We henceforth denote the density associated with the distribution $F$ defined in Eq. (5) by $f$.

Proposition 4 (Feasibility of the equilibrium distribution). There exists a stopping strategy inducing the distribution $F$ defined in Eq. (5).

Proof. To verify the condition in Pedersen and Peskir [15], we need a process which starts at zero. Thus, we consider the process $\tilde{X}_{t}=X_{t}-X_{0}$. After some transformations, we get $S(x-$ $\left.x_{0}\right)=-\frac{\sigma^{2}}{2 \mu}\left(1-\exp \left(\frac{2 \mu x_{0}}{\sigma^{2}}\right)\right)(\phi(x)-1)$. This gives us

$$
\begin{aligned}
m & =\int_{\mathbb{R}} S\left(x-x_{0}\right) f(x) \mathrm{d} x \\
& =-\frac{\sigma^{2}}{2 \mu}\left(1-\exp \left(\frac{2 \mu x_{0}}{\sigma^{2}}\right)\right)\left(\int_{\mathbb{R}} f(x) \phi(x) \mathrm{d} x-1\right) .
\end{aligned}
$$

\footnotetext{
${ }^{2}$ A real-valued process $\left(X_{t}\right)_{t \in \mathbb{R}_{+}}$is a non-singular diffusion if it solves the stochastic differential equation $\mathrm{d} X_{t}=$ $\mu\left(X_{t}\right) \mathrm{d} t+\sigma\left(X_{t}\right) \mathrm{d} B_{t}$, with $\sigma(x)>0$ for all $x \in \mathbb{R}$ and $B$ is a Brownian motion. Hence, the Brownian motion with drift defined in Eq. (1) is a special case of a non-singular diffusion.

${ }^{3}$ Pedersen and Peskir define the scale function $S$ for general non-singular diffusions by $S(x)=$ $\int_{0}^{x} \exp \left(-2 \int_{0}^{u} \frac{\mu(r)}{\sigma^{2}(r)} \mathrm{d} r\right) \mathrm{d} u$. As $\mu$ and $\sigma$ are constant in our model, the scale function simplifies to Eq. (8).
} 
Consequently, to obtain $m=0$, it remains to show $\int_{\mathbb{R}} f(x) \phi(x) \mathrm{d} x=1$.

$$
\begin{aligned}
\int_{\mathbb{R}} f(x) \phi(x) \mathrm{d} x & =\int_{0}^{\bar{x}} \frac{\left(n^{-\frac{1}{n-1}}\right)}{n-1} \phi(x)^{-\frac{n-2}{n-1}} \phi^{\prime}(x) \phi(x) \mathrm{d} x \\
& =\int_{\phi(0)}^{\phi(\bar{x})} \frac{\left(n^{-\frac{1}{n-1}}\right)}{n-1} y^{\frac{1}{n-1}} \mathrm{~d} y \\
& =\left[\frac{\left(n^{-\frac{1}{n-1}}\right)}{n} y^{\frac{n}{n-1}}\right]_{y=\phi(0)=0}^{y=\phi(\bar{x})=n} \\
& =1 .
\end{aligned}
$$

As $\int_{\mathbb{R}} f(x) \phi(x) \mathrm{d} x=1$, we obtain $m=0$ from Eq. (9). By Theorem 2.1 in Pedersen and Peskir [15], there exists a stopping strategy inducing the distribution $F$ defined in Eq. (5).

The equilibrium distribution in the present paper is similar to that of an all-pay auction with complete information (e.g., Hillman and Samet [8], or Baye et al. [2]). ${ }^{4}$ In both settings, the joint equilibrium distribution of the other players makes each player indifferent. The trade-off between a higher risk and a higher chance to win the prize thus serves as an implicit cost.

In the static two-player contest of Lazear and Rosen [11], contest success depends on the effort choice and the realization of a random variable. In their framework, contests are suitable to induce the optimal effort decisions. If, in our two-player model, agents had to specify a fixed date at which they stop, they would stop immediately for negative values of the drift. Hence, the dynamic nature of the decision problem for each player drives our results.

\section{Extension to the asymmetric two-player case}

In this section, we extend our equilibrium construction to the case of two players $i \in\{1,2\}$ whose processes $\left(X_{t}^{i}\right)_{t \in \mathbb{R}_{+}}$differ in starting value $x_{0}^{i}$, drift $\mu_{i}$ and variance $\sigma_{i}$, i.e.,

$$
X_{t}^{i}=x_{0}^{i}+\mu_{i} t+\sigma_{i} B_{t}^{i} .
$$

Intuitively, the solution procedure parallels the symmetric case in that both players are indifferent between any stopping strategy on $(0, \bar{x}]$. However, the weaker player now places a mass point at zero to satisfy the feasibility condition. Similar to Assumption 1, we impose an upper bound on the drift $\mu_{i}$ :

Assumption 2. The drift of both players is negative, i.e., $\mu_{1}<0$ and $\mu_{2}<0$.

Define the transformation $\psi_{i}(x)=\exp \left(\frac{-2 \mu_{i} x}{\sigma_{i}^{2}}\right)$ similar to Eq. (6). In the following, we construct feasible distributions and find $\bar{x} \in \mathbb{R}_{+}$such that both players are indifferent between any strategy that stops with probability one on the interval $(0, \bar{x}]$. If the distribution $F_{i}$ is of the form

\footnotetext{
4 Complete information about valuations in the all-pay auction corresponds to complete information about starting values in this paper.
} 


$$
F_{i}\left(x, \alpha_{i}, \bar{x}^{i}\right)=\min \left\{1, \alpha_{i}+\left(1-\alpha_{i}\right) \frac{\psi_{j}(x)-1}{\psi_{j}\left(\bar{x}^{i}\right)-1}\right\}
$$

for some $\alpha_{i} \in[0,1]$, agent $j$ is indifferent between all stopping strategies that stop with probability one on the interval $\left(0, \bar{x}^{i}\right]$, because $\left(\psi_{j}\left(X_{t}^{j}\right)\right)_{t \in \mathbb{R}_{+}}$is a martingale. We construct an equilibrium in which both agents use distributions of the form in Eq. (10) and at least one agent places no mass point at zero, i.e., $\alpha_{i}=0$ or $\alpha_{j}=0$.

The following result applies Lemma 1 to the asymmetric setting. It provides a sufficient condition for the feasibility of a distribution $F_{i}$.

Lemma 2 (Feasibility). There exists a stopping time $\tau_{i}$ inducing a distribution $F_{i}$ of agent $i$ if

$$
\psi_{i}\left(x_{0}^{i}\right)=\int \psi_{i}(x) \mathrm{d} F_{i}(x) .
$$

If player $i$ places no mass point at zero, Eq. (11) becomes $\psi_{i}\left(x_{0}^{i}\right)=\int_{0}^{\bar{x}^{i}} f_{i}(x) \psi_{i}(x) \mathrm{d} x$. In Appendix A, we show that there exist unique $\bar{x}^{1}>x_{0}^{1}$ and $\bar{x}^{2}>x_{0}^{2}$ which satisfy

$$
\psi_{i}\left(x_{0}^{i}\right)=\int_{0}^{\bar{x}^{i}} f_{i}\left(x, 0, \bar{x}^{i}\right) \psi_{i}(x) \mathrm{d} x .
$$

Assumption 3. Without loss of generality, assume $\bar{x}^{1} \geqslant \bar{x}^{2}$.

Set $\bar{x}=\bar{x}^{1}$. By construction, $F_{1}(\cdot, 0, \bar{x})$ is a distribution and feasible for agent 1 . Agent 2 places a mass point of size $\alpha_{2} \geqslant 0$ at zero such that the distribution $F_{2}\left(\cdot, \alpha_{2}, \bar{x}\right)$ is feasible for agent 2. By Lemma 2, $F_{2}$ is feasible if it satisfies Eq. (11), i.e., $\alpha_{2}$ solves the linear equation

$$
\begin{aligned}
\psi_{2}\left(x_{0}^{2}\right) & =F_{2}\left(0, \alpha_{2}, \bar{x}\right) \psi_{2}(0)+\int_{0}^{\bar{x}} f_{2}\left(x, \alpha_{2}, \bar{x}\right) \psi_{2}(x) \mathrm{d} x \\
& =\alpha_{2}+\left(1-\alpha_{2}\right) \int_{0}^{\bar{x}} f_{2}(x, 0, \bar{x}) \psi_{2}(x) \mathrm{d} x .
\end{aligned}
$$

As $\bar{x} \geqslant \bar{x}^{2}$, we obtain $\int_{0}^{\bar{x}} f_{2}(x, 0, \bar{x}) \psi_{2}(x) \mathrm{d} x \geqslant \psi_{2}\left(x_{0}^{2}\right)>1$. Therefore, Eq. (13) has a unique solution $\alpha_{2} \in[0,1]$.

Proposition 5 (Asymmetric equilibrium distributions). There exists a Nash equilibrium in which the equilibrium distributions $F_{1}$ and $F_{2}$ satisfy Eq. (10) with $\alpha_{1}=0, \bar{x}$ is the solution of Eq. (12) for player 1, and $\alpha_{2}$ is the solution to Eq. (13).

Proof. The distributions $F_{1}$ and $F_{2}$ are feasible since they satisfy Eq. (11) by construction. Moreover, each player is indifferent between any strategy that stops with probability one on the support $[0, \bar{x}]$. Hence, following the arguments from Proposition 1, for any stopping time $\tau^{i}$, we obtain

$$
\mathbb{E}\left(u_{i}\left(X_{\tau^{i}}^{i}\right)\right) \leqslant u_{i}\left(x_{0}\right)=\mathbb{E}\left(u_{i}\left(X_{\tau_{*}^{i}}^{i}\right)\right),
$$

where $\tau_{*}^{i}$ is a strategy that induces the equilibrium distribution of player $i$. 
For heterogeneity in the starting values only-without loss of generality $x_{0}^{1}>x_{0}^{2}$ - this solution procedure yields the following result:

Proposition 6. In equilibrium, the cdf of the first player is

$$
F_{1}(x)=\min \left\{1, \frac{1}{2} \frac{\exp \left(\frac{-2 \mu x}{\sigma^{2}}\right)-1}{\exp \left(\frac{-2 \mu x_{0}^{1}}{\sigma^{2}}\right)-1}\right\} \text {. }
$$

The cdf of the second player is

$$
F_{2}(x)=\min \left\{1,\left(1-\rho\left(0, x_{0}^{1}, x_{0}^{2}\right)\right)+\rho\left(0, x_{0}^{1}, x_{0}^{2}\right) \frac{1}{2} \frac{\exp \left(\frac{-2 \mu x}{\sigma^{2}}\right)-1}{\exp \left(\frac{-2 \mu x_{0}^{1}}{\sigma^{2}}\right)-1}\right\} .
$$

An equilibrium strategy for player 2 is to first play until $X_{t}^{2} \notin\left(0, x_{0}^{1}\right)$; then use the same stopping strategy as player 1 if he reaches $x_{0}^{1}$. This strategy induces the above distribution, where the constant $1-\rho\left(0, x_{0}^{1}, x_{0}^{2}\right)$ is the probability of absorption in zero.

Compared to the symmetric case, the player with the lower starting value takes more risks here. In particular, he loses everything with probability $1-\rho\left(0, x_{0}^{1}, x_{0}^{2}\right)$ and takes the same gamble as player 1 with probability $\rho\left(0, x_{0}^{1}, x_{0}^{2}\right)$. Asymmetry in the contest leads to higher percentage losses for a negative drift, because the handicapped player takes higher risks to compensate his initial disadvantage.

\section{Comparative statics}

This section analyzes how changes in the parameters affect the expected value of the stopped processes. To determine the expected value, we first calculate the density from the cdf defined in Eq. (5):

$$
f(x)=\frac{2 \mu}{n(n-1) \sigma^{2}} \sqrt[\frac{2-n}{n-1}]{\frac{\exp \left(\frac{-2 \mu x}{\sigma^{2}}\right)-1}{n\left(\exp \left(\frac{-2 \mu x_{0}}{\sigma^{2}}\right)-1\right)}} \frac{\exp \left(\frac{-2 \mu x}{\sigma^{2}}\right)}{1-\exp \left(\frac{-2 \mu x_{0}}{\sigma^{2}}\right)} .
$$

In what follows, we restrict attention to the two-player case for tractability. We use the density $f$ to derive the expected value of the stopped processes in the unique equilibrium for two players:

$$
\begin{aligned}
\mathbb{E}\left(X_{\tau}\right) & =\int_{0}^{\bar{x}} x f(x) \mathrm{d} x \\
& =\frac{\sigma^{2}}{2 \mu}+\left(1+\frac{1}{2\left(\exp \left(-\frac{2 \mu x_{0}}{\sigma^{2}}\right)-1\right)}\right)\left(x_{0}-\frac{\sigma^{2} \log \left(2-\exp \left(\frac{2 \mu x_{0}}{\sigma^{2}}\right)\right)}{2 \mu}\right) .
\end{aligned}
$$

The explicit formula of the expected value allows us to characterize its shape in the following proposition.

Proposition 7 (Comparative statics). $\mathbb{E}\left(X_{\tau}\right)$ is quasi-convex in $\mu$, falling and then rising. If $\mu<0, \mathbb{E}\left(X_{\tau}\right)$ is quasi-convex in $\sigma$, falling and then rising. $\mathbb{E}\left(X_{\tau}\right)$ attains its minimum at an intermediate negative drift level $-\infty<\mu<0$. (See Figs. 2, 3.) 


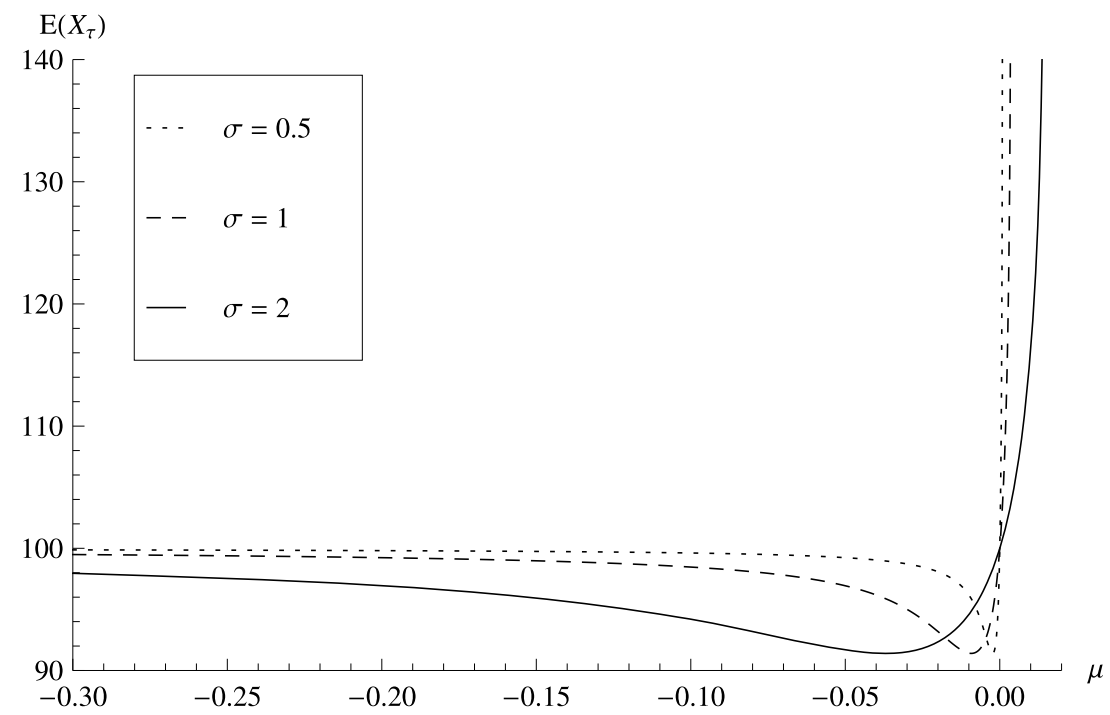

Fig. 2. An example $\left(n=2, x_{0}=100\right)$ of the expected value of the stopped processes $\mathbb{E}\left(X_{\tau}\right)$ depending on the drift $\mu$ for different values of variance $\sigma$.

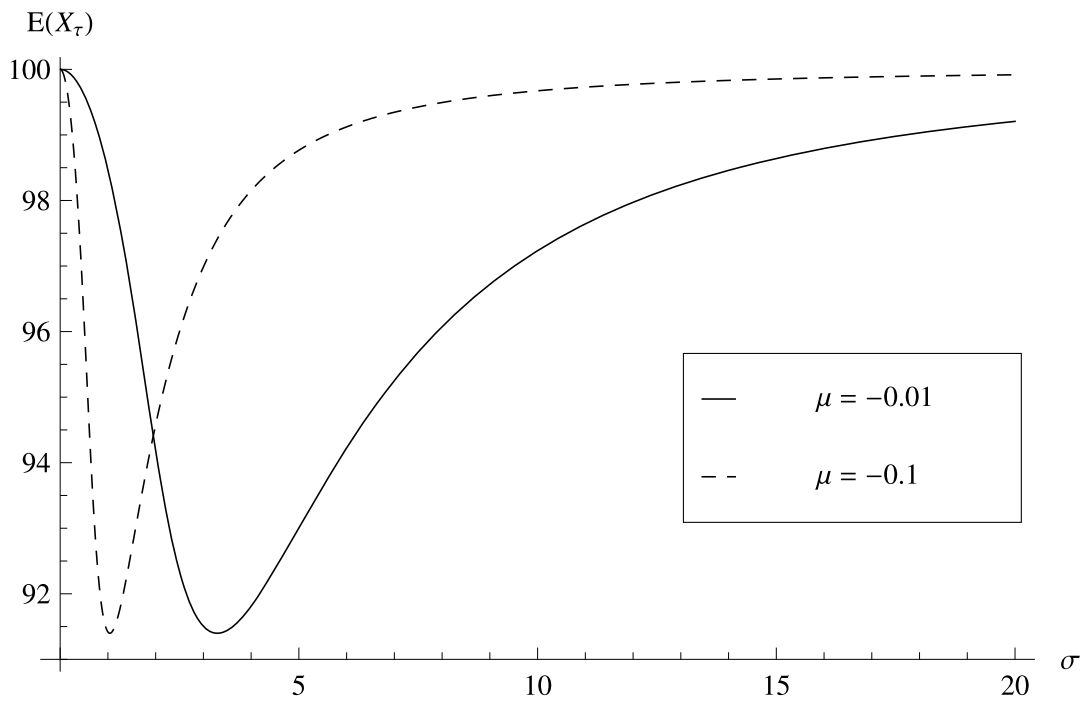

Fig. 3. An example $\left(n=2, x_{0}=100\right)$ of the expected value of the stopped processes $\mathbb{E}\left(X_{\tau}\right)$ depending on the variance $\sigma$ for different values of drift $\mu$.

Curiously, even though the expected net gains per time obviously rise in the drift $\mu$, the expected value of the stopped process does not always rise for $\mu<0$. The reason is that the equilibrium expected stopping time increases in the drift. Similarly, for $\mu<0$, the effect of an increase in the variance on the expected value of the stopped process is ambiguous. With zero variance, both players would stop immediately because of the negative drift. As the variance tends to infinity, players would also stop almost immediately, since it requires very little time to 
induce the equilibrium distribution due to the large variance. Thus, the expected stopping time and thereby the loss in expected value is maximal at an intermediate level of variance.

From an economic point of view, Proposition 7 illustrates a drawback of relative performance payments in risky environments: even if risky investment opportunities have only a slightly negative expected value, the principal might lose a lot in expectation. Intuitively, contestants only care about outperforming each other and thus behave as if they were risk-loving. A simple linear compensation scheme based on absolute performance would avoid this drawback.

\section{Conclusion}

We have studied a new continuous time model of contests. Contrary to the previous literature, players trade off a higher variance against a lower expected value in equilibrium. If there are no good investment opportunities available, e.g., in a declining industry, contestants thus behave as if they were risk-loving - they invest in projects with negative expected returns. According to our main characterization result, Proposition 7, this problem is most severe for an intermediate negative value of the drift.

From a technical point of view, this paper has developed a new method to verify equilibrium existence. The approach via Skorokhod embeddings seems promising to analyze other models without observability, because there are many sufficient conditions available in the probability theory literature.

\section{Acknowledgments}

We would like to thank Paul Heidhues, Benny Moldovanu, and Tymon Tatur for their enduring support of this work. We are grateful to an anonymous associate editor and an anonymous referee whose comments and suggestions have greatly improved the quality of the paper. Furthermore, we are grateful to Dirk Bergemann, Norbert Christopeit, Faruk Gul, Sergei Izmalkov, Georg Kirchsteiger, Matthias Lang, Stephan Lauermann, Moritz Meyer-ter-Vehn, Sven Rady, Frank Riedel, Xianwen Shi, Nora Szech, Philipp Weinschenk, and many others for helpful discussions. Financial support from the Bonn Graduate School of Economics, German Science Foundation, Hausdorff Center for Mathematics, and SFB TR 15 is gratefully acknowledged.

\section{Appendix A}

Proof of Proposition 3. First note that any equilibrium distribution is continuous, i.e., no player places a mass point above zero. We omit the proof since it is simply a specialization of now standard arguments in static game theory with a continuous state space; e.g., Burdett and Judd [3].

Denote by $\left(\tilde{F}_{i}, \tilde{F}_{j}\right)$ the distributions in an equilibrium and by $F$ the equilibrium distribution defined in Eq. (5). Denote the associated winning probabilities by $\left(\tilde{u}_{j}, \tilde{u}_{i}\right)$ and $\left(u_{j}, u_{i}\right)$. Define $\bar{x}=\sup \{x: F(x)<1\}$.

By the minmax property for two-player constant sum games, any strategy inducing the equilibrium distribution $F$ is a best response for each player in any equilibrium. Furthermore, any equilibrium strategy is a best response against the distribution $F$.

For all stopping times $\tau^{j}>t, \mathbb{E}\left(u_{j}\left(X_{\tau^{j}}^{j}\right) \mid X_{t}^{j}=\bar{x}\right)<u_{j}(\bar{x})=1$, since $u_{j}(\bar{x}-\epsilon)<1$ for all $\epsilon>0$. Hence, a player strictly prefers to stop at $\bar{x}$ in any equilibrium, which implies $\tilde{F}_{i}(\bar{x})=$ $\tilde{F}_{j}(\bar{x})=1$. 
As $F$ is an equilibrium distribution, there exists no feasible distribution $\tilde{F}_{i}$ that first-order stochastically dominates $F$. Hence, for any equilibrium distribution $\tilde{F}_{i} \neq F$, there exists an $x \in$ $(0, \bar{x})$ such that $F(x)>\tilde{F}_{i}(x)$ and $\tilde{u}_{j}(x)<u_{j}(x)$. As $\tilde{F}_{i}$ is continuous at $\bar{x}$, i.e., $\tilde{u}_{j}(\bar{x})=1$, we obtain

$$
\begin{aligned}
\tilde{u}_{j}(x)<u_{j}(x) & =\rho(0, \bar{x}, x) \leqslant \rho(0, \bar{x}, x)+(1-\rho(0, \bar{x}, x)) \tilde{u}_{j}(0) \\
& =\mathbb{E}\left(\tilde{u}_{j}\left(X_{\tau_{(0, \bar{x})}^{j}}^{j}\right) \mid X_{t}^{j}=x\right) .
\end{aligned}
$$

By continuity of $\tilde{u}_{j}$ at $x$, it is not optimal for player $j$ to stop in some neighborhood of $x$. As $F$ places positive mass in any neighborhood of $x, F$ is not a best response against $\tilde{F}_{i}$. Thus, $\tilde{F}_{i}$ is not an equilibrium distribution.

Proof of Lemma 2. By Lemma $1, \int_{\mathbb{R}} S\left(x-x_{0}^{i}\right) \mathrm{d} F_{i}(x)=0$ is a sufficient condition for feasibility which yields

$$
0=\int_{\mathbb{R}} S\left(x-x_{0}^{i}\right) \mathrm{d} F_{i}(x) \mathrm{d} x=\mathbb{E}\left(S\left(X_{\tau^{i}}^{i}-x_{0}^{i}\right)\right)=\mathbb{E}\left(-\frac{\sigma_{i}^{2}}{2 \mu_{i}}\left(\psi\left(X_{\tau^{i}}^{i}-x_{0}^{i}\right)-1\right)\right) .
$$

Thus, to show the existence of a strategy $\tau^{i}$ inducing a distribution $F_{i}$, it is sufficient to verify that $\mathbb{E}\left(\psi\left(X_{\tau^{i}}^{i}\right)\right)=\psi\left(x_{0}^{i}\right)$.

Lemma 3. Eq. (12) has a unique solution $\bar{x}^{i}$.

Proof. As $\int_{0}^{\bar{x}^{i}} f_{i}\left(x, 0, \bar{x}^{i}\right) \psi_{i}(x) \mathrm{d} x$ is strictly increasing in $\bar{x}^{i}$, it suffices to show existence of an $\bar{x}^{i}$ satisfying the following equation

$$
\begin{aligned}
\psi_{i}\left(x_{0}^{i}\right)= & \int_{0}^{\bar{x}^{i}} f_{i}\left(x, 0, \bar{x}^{i}\right) \psi_{i}(x) \mathrm{d} x=\int_{0}^{\bar{x}^{i}} \frac{\psi_{j}^{\prime}(x)}{\psi_{j}\left(\bar{x}^{i}\right)-1} \psi_{i}(x) \mathrm{d} x \\
\Rightarrow \quad 0 & =\int_{0}^{\bar{x}^{i}} \psi_{j}^{\prime}(x)\left(\psi_{i}(x)-\psi_{i}\left(x_{0}^{i}\right)\right) \mathrm{d} x .
\end{aligned}
$$

Since $\mu_{i}<0, \psi_{j}^{\prime}(x)\left(\psi_{i}(x)-\psi_{i}\left(x_{0}^{i}\right)\right)<0$ for all $x<x_{0}$. Moreover, both $\left(\psi_{i}(x)-\psi_{i}\left(x_{0}^{i}\right)\right)$ and $\psi_{j}^{\prime}(x)$ are positive and bounded away from zero for all $x>x_{0}^{i}$. By continuity and the intermediate value theorem, there exists an $\bar{x}^{i}>x_{0}^{i}$ satisfying Eq. (14).

Proof of Proposition 6. This proof mimics the construction in the main text. We first consider Eq. (12) for $\mu_{1}=\mu_{2}=\mu$ and $\sigma_{1}=\sigma_{2}=\sigma$ :

$$
\exp \left(\frac{-2 \mu x_{0}^{i}}{\sigma^{2}}\right)=\int_{0}^{\bar{x}^{i}}-\frac{2 \mu}{\sigma^{2}} \frac{\exp \left(\frac{-4 \mu x}{\sigma^{2}}\right)}{\exp \left(\frac{-2 \mu \bar{x}^{i}}{\sigma^{2}}\right)-1} \mathrm{~d} x
$$

Solving for $\bar{x}^{i}$ yields $\bar{x}^{i}=-\frac{\sigma^{2}}{2 \mu} \log \left(2 \exp \left(\frac{-2 \mu x_{0}^{i}}{\sigma^{2}}\right)-1\right)$. Since $x_{0}^{1}>x_{0}^{2}$ and $\mu<0$ by Assumption 2, we obtain $\bar{x}^{1}>\bar{x}^{2}$. We define $\bar{x}=\bar{x}^{1}$. We solve Eq. (13) to obtain the claim $\alpha_{2}=1-\rho\left(0, x_{0}^{1}, x_{0}^{2}\right)$ : 


$$
\begin{aligned}
\exp \left(\frac{-2 \mu x_{0}^{2}}{\sigma^{2}}\right)=\alpha_{2}+\left(1-\alpha_{2}\right) \int_{0}^{\bar{x}}-\frac{2 \mu}{\sigma^{2}} \frac{\exp \left(\frac{-4 \mu x}{\sigma^{2}}\right)}{\exp \left(\frac{-2 \mu \bar{x}}{\sigma^{2}}\right)-1} \mathrm{~d} x \\
\Rightarrow \alpha_{2}=\frac{\exp \left(\frac{-2 \mu x_{0}^{2}}{\sigma^{2}}\right)-\frac{1}{2}\left(\exp \left(\frac{-2 \mu \bar{x}}{\sigma^{2}}\right)+1\right)}{1-\frac{1}{2}\left(\exp \left(\frac{-2 \mu \bar{x}}{\sigma^{2}}\right)+1\right)} \\
=\frac{\exp \left(\frac{-2 \mu x_{0}^{2}}{\sigma^{2}}\right)-\exp \left(\frac{-2 \mu x_{0}^{1}}{\sigma^{2}}\right)}{1-\exp \left(\frac{-2 \mu x_{0}^{1}}{\sigma^{2}}\right)}=1-\rho\left(0, x_{0}^{1}, x_{0}^{2}\right)
\end{aligned}
$$

Proof of Proposition 7. We apply the monotone transformation $y=\exp \left(\frac{2 \mu x_{0}}{\sigma^{2}}\right)$ to $\mathbb{E}\left(X_{\tau}\right)$ to get

$$
\begin{aligned}
\mathbb{E}\left(X_{\tau}\right) & =\frac{x_{0}}{\log (y)}+\left(1+\frac{y}{2(1-y)}\right)\left(x_{0}-\frac{x_{0} \log (2-y)}{\log (y)}\right) \\
& =x_{0}\left(\frac{1}{\log (y)}+\left(1+\frac{y}{2(1-y)}\right)\left(1-\frac{\log (2-y)}{\log (y)}\right)\right)
\end{aligned}
$$

for $y \neq 1$. This expression is convex if and only if it is convex for $x_{0}=1$. Assumption 1 implies $y \in(0,2)$.

$$
\begin{aligned}
\frac{\partial^{2} \mathbb{E}\left(X_{\tau}\right) / x_{0}}{\partial y^{2}}= & \frac{4(-2+y)(-1+y)^{3}+2(-1+y)^{2}\left(2-5 y+2 y^{2}\right) \log (y)}{2(-2+y)(-1+y)^{3} y^{2} \log (y)^{3}} \\
& +\frac{y^{2}\left(3-4 y+y^{2}\right) \log (y)^{2}-2(-2+y) y^{2} \log (y)^{3}}{2(-2+y)(-1+y)^{3} y^{2} \log (y)^{3}} \\
& -\frac{(-2+y) \log (2-y)\left(2(-2+y)(-1+y)^{2}-2 y^{2} \log (y)^{2}\right)}{2(-2+y)(-1+y)^{3} y^{2} \log (y)^{3}} \\
& -\frac{(-2+y) \log (2-y) \log (y)\left(-2+7 y-6 y^{2}+y^{3}\right)}{2(-2+y)(-1+y)^{3} y^{2} \log (y)^{3}}
\end{aligned}
$$

with the continuous extension $\frac{\partial^{2} \mathbb{E}\left(X_{\tau}\right) / x_{0}}{\partial y^{2}}=\frac{1}{6}$ at $y=1$. Simple algebra shows that nominator and denominator are negative on $y \in(0,2), y \neq 1$. Hence, the function is convex on $(0,2)$. As $y$ is monotone increasing in $\mu, \mathbb{E}\left(X_{\tau^{i}}^{i}\right)$ is quasi-convex in $\mu$. As $y$ is also monotone increasing (decreasing) in $\sigma$ for $\mu<0(\mu>0), \mathbb{E}\left(X_{\tau^{i}}^{i}\right)$ is quasi-convex (quasi-concave) in $\sigma$ if $\mu<0$ $(\mu>0)$.

We now show that $\mathbb{E}\left(X_{\tau}\right)$ is first decreasing, then increasing. For $\mu \rightarrow-\infty$ and $\mu \rightarrow 0$, $\mathbb{E}\left(X_{\tau}\right) \rightarrow x_{0}$. For any negative value of $\mu$, the expected value of the stopped process is smaller than $x_{0}$, because the process is a supermartingale. Hence, by quasi-convexity, $\mathbb{E}\left(X_{\tau}\right)$ has to be first decreasing, then increasing.

It remains to show that the minimum of $\mathbb{E}\left(X_{\tau}\right)$ is attained for $-\infty<\mu<0$. By Doob's optional stopping theorem, for any $\mu \geqslant 0$ and any stopping time $\tau$, we have $\mathbb{E}\left(X_{\tau}\right) \geqslant X_{0}$. On the other hand, for any $\mu<0$ and any stopping time $\tau$, we have $\mathbb{E}\left(X_{\tau}\right) \leqslant X_{0}$. Since $\mathbb{E}\left(X_{\tau}\right)$ is quasi-convex in $\mu$, first decreasing and then increasing, we obtain that $\mathbb{E}\left(X_{\tau}\right)$ is minimized for some value $-\infty<\mu<0$. 


\section{References}

[1] A. Anderson, L.M.B. Cabral, Go for broke or play it safe? Dynamic competition with choice of variance, RAND J. Econ. 38 (2007) 593-609.

[2] M.R. Baye, D. Kovenock, C. de Vries, The all-pay auction with complete information, Econ. Theory 8 (1996) 291-305.

[3] K. Burdett, K.L. Judd, Equilibrium price dispersion, Econometrica 51 (1983) 955-969.

[4] G.W. Downs, D.M. Rocke, Conflict, agency, and gambling for resurrection: The principal-agent problem goes to war, Amer. J. Polit. Sci. 38 (1994) 362-380.

[5] D. Fudenberg, J. Tirole, A theory of exit in duopoly, Econometrica 54 (1986) 943-960.

[6] F. Gul, W. Pesendorfer, The war of information, Rev. Econ. Stud. 79 (2012) 707-734.

[7] C. Harris, J. Vickers, Racing with uncertainty, Rev. Econ. Stud. 54 (1987) 1-21.

[8] A.L. Hillman, D. Samet, Dissipation of contestable rents by small numbers of contenders, Public Choice 54 (1987) 63-82.

[9] H. Hvide, Tournament rewards and risk taking, J. Lab. Econ. 20 (2002) 877-898.

[10] S. Karlin, Reduction of certain classes of games to integral equations, in: H. Kuhn, A. Tucker (Eds.), Contributions to the Theory of Games, II, in: Ann. Math. Stud., vol. 28, Princeton University Press, Princeton, 1953, pp. $125-128$.

[11] E.P. Lazear, S. Rosen, Rank-order tournaments as optimum labor contracts, J. Polit. Economy 89 (1981) $841-864$.

[12] G. Moscarini, L. Smith, Optimal dynamic contests, Working paper, 2007.

[13] J. Oblój, The Skorokhod embedding problem and its offspring, Probab. Surv. 1 (2004) 321-392.

[14] A. Park, L. Smith, Caller number five and related timing games, Theoretical Econ. 3 (2008) 231-256.

[15] J.L. Pedersen, G. Peskir, The Azéma-Yor embedding in non-singular diffusions, Stoch. Process. Their Appl. 96 (2001) 305-312.

[16] D. Revuz, M. Yor, Continuous Martingales and Brownian Motion, Springer Verlag, 2005.

[17] A.V. Skorokhod, Issledovaniya po teorii sluchainykh protsessov (Stokhasticheskie differentsialnye uravneniya i predelnye teoremy dlya protsessov Markova), Izdat Kiev University, 1961.

[18] A.V. Skorokhod, Studies in the Theory of Random Processes, Addison-Wesley Publishing Co., 1965, translated from the Russian by Scripta Technica, Inc. 